\title{
Supplementary Material: Contrasting biosphere responses to hydrometeorological extremes: revisiting the 2010 western Russian Heatwave
}

\author{
Milan Flach ${ }^{1}$, Sebastian Sippel ${ }^{2}$, Fabian Gans $^{1}$, Ana Bastos ${ }^{3}$, Alexander Brenning ${ }^{4,5}$, \\ Markus Reichstein ${ }^{1,5}$, and Miguel D. Mahecha ${ }^{1,5}$ \\ ${ }^{1}$ Max Planck Institute for Biogeochemistry, Department Biogeochemical Integration, P. O. Box 1001 64, D-07701 Jena, \\ Germany \\ ${ }^{2}$ Norwegian Institute of Bioeconomy Research, Ås, Norway \\ ${ }^{3}$ Laboratoire des Sciences du Climat et de l'Environnement, LSCE/IPSL, CEA-CNRS-UVSQ, Université Paris-Saclay, 91191 \\ Gif-sur-Yvette, France \\ ${ }^{4}$ Friedrich Schiller University Jena, Department of Geography, Jena, Germany \\ ${ }^{5}$ Michael Stifel Center Jena for Data-driven and Simulation Science, Jena, Germany
}

Correspondence: Milan Flach (milan.flach@bgc-jena.mpg.de)

\section{S1 Overlapping Spatial Segmentation}

Extreme anomalies are usually more perceived in the context of some similar region instead of completely locally (in each grid cell) or globally. Thus, for quantifying the relative effect of anomalies on the biosphere, we identify segments with similar climate and ecosystem patterns independent of their extremes according to Mahecha et al. (2017). We compute the median seasonal cycles (MSC) and the cycle in the median absolute deviation (as guess for the variance) for each variable separately. We sort the seasonal cycles of all variables according to the permutation of temperature to remove effects of similar, but only lagged seasonal cycles. We concatenate seasonal cycles of all variables. Subsequently, we train a Principal Component Analysis (PCA) on 5000 spatial samples of this matrix and project the principal component loadings for each grid cell. We obtain similar seasonal cycles by the dividing the PC-Hyperspace into cubes with equal edge length. Two choices have to be made: The dimensionality, i.e. number of principal components $\left(N_{P C}\right)$ and the edge length of the (hyper)cubes $(a)$. $N_{P C}$ presents a trade-off between more accurate information (many dimensions) and too high dimensionality which would result in many spatial segments containing only one gridcell. We choose $N_{P C}=4$ which explains already $71 \%$ of the mean seasonal cycles variance. The edge length $a$ is chosen in such a way, that 1 ) it is sufficiently small (to get only very similar seasonal cycles) and that 2) gridcells are not singled out without any other similar gridcell. The similarities in seasonality which we obtain by this procedure are somehow plausible from a climate classification and ecosystems perspective (Fig. S1 1). However, please note that the goal of the spatial segmentation is not to obtain a perfect classification into different ecosystems, but rather to provide small (overlapping) spatial segments with few gridcells of similar seasonality. 


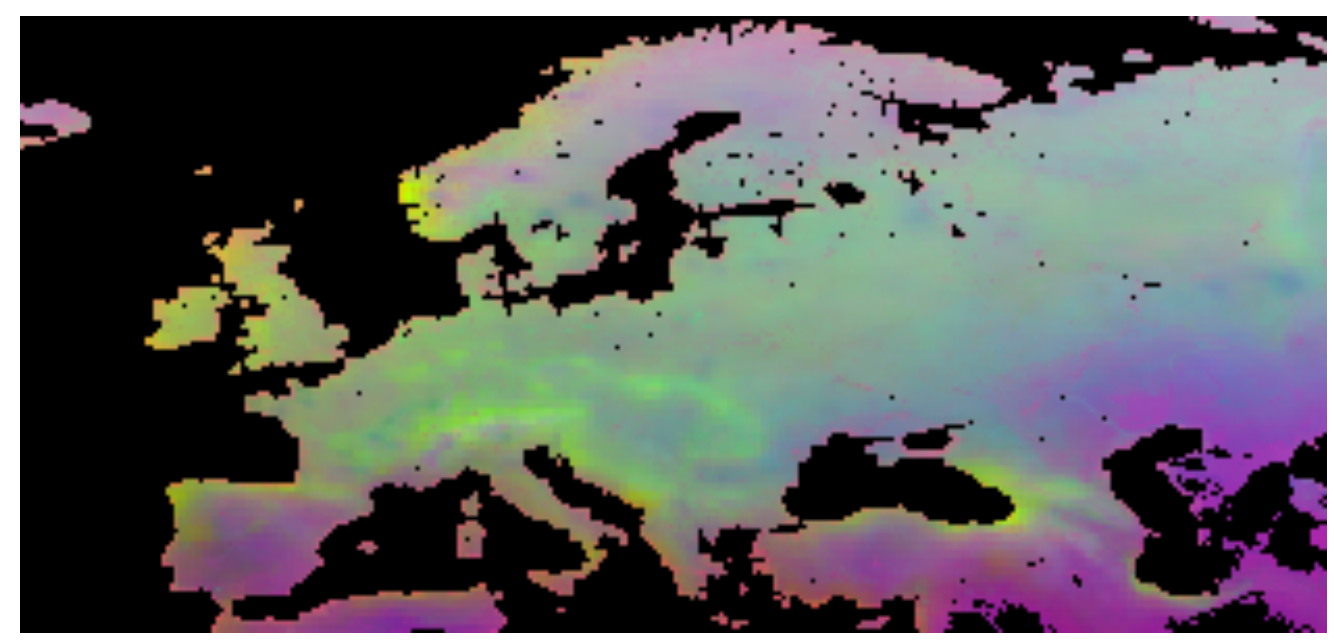

Figure S1 1. Map of the first three principal components of the seasonal cycles in the median and the variance. The principal components are visualized as a red (R), green $(\mathrm{G})$, and blue (B) channels of an image. Similar colours indicate similar mean patterns in the biosphere and hydrometeorological variables. This information is used to define overlapping regions with similar mean patterns for anomaly detection.

\section{S2 Temporal Evolution of the Heatwave}

In 2010, western Russia was hit by two hydrometeorological anomalies (Fig. S2 2). The multivariate spring anomaly of the hydrometeorological variables starts at the beginning of May (Longitude $=30.25-60.0^{\circ} \mathrm{E}$, Latitude $\geq 55^{\circ} \mathrm{N}$ ). The summer heatwave is usually referred to as Russian Heatwave in the hydrometeorological variables. It starts in the East in the beginning of June and develops a constant extent from mid June until it starts to vanish in mid August (Longitude $=28.75$ to $60.25^{\circ} \mathrm{E}$, Latitude $=48.25$ to $66.75^{\circ} \mathrm{N}$ ). Both multivariate hydrometeorological anomalies (spring and summer) have a corresponding multivariate biosphere anomaly. The biosphere anomaly in western Russia started in the beginning of May 2010 (May, 9th and May, 15th, Fig. S2 3) for Northern latitudes $\left(>55^{\circ} \mathrm{N}\right)$. After a short break it continued in South Russia $\left(<57^{\circ} \mathrm{N}\right)$ in the beginning of June and persisted more than 3 month until Mid August (August, 13th, Fig. S2 3, Latidude $=47.75-68.75^{\circ} \mathrm{N}$, Longitude $=30.25-60.0^{\circ} \mathrm{E}$ ) 

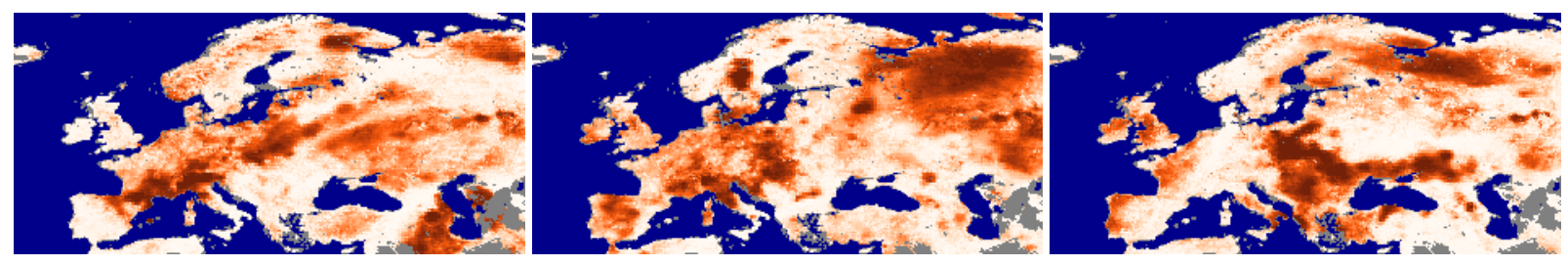

d 2010-05-25

e 2010-06-02

f 2010-06-10
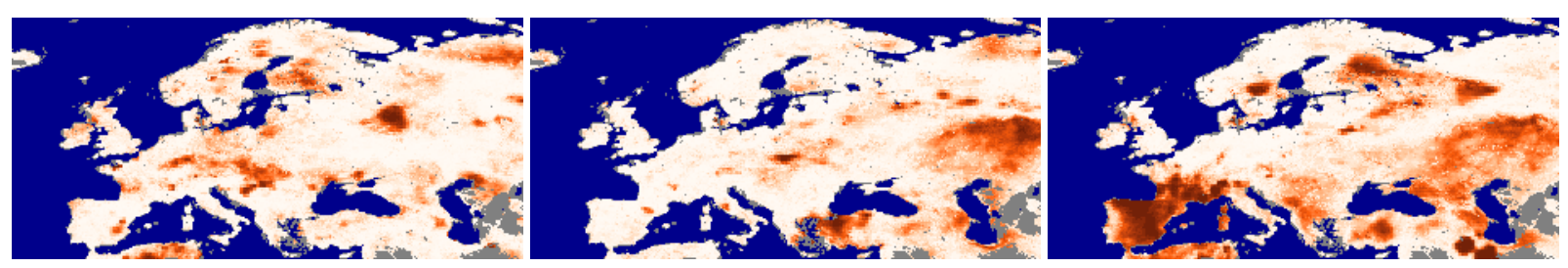

g 2010-06-18

h 2010-06-26

i 2010-07-04
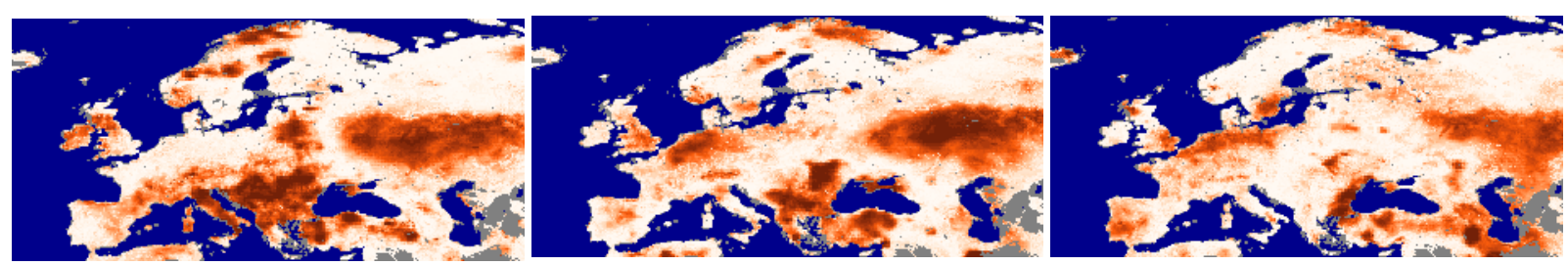

\section{j 2010-07-12}

k 2010-07-20

1 2010-07-28
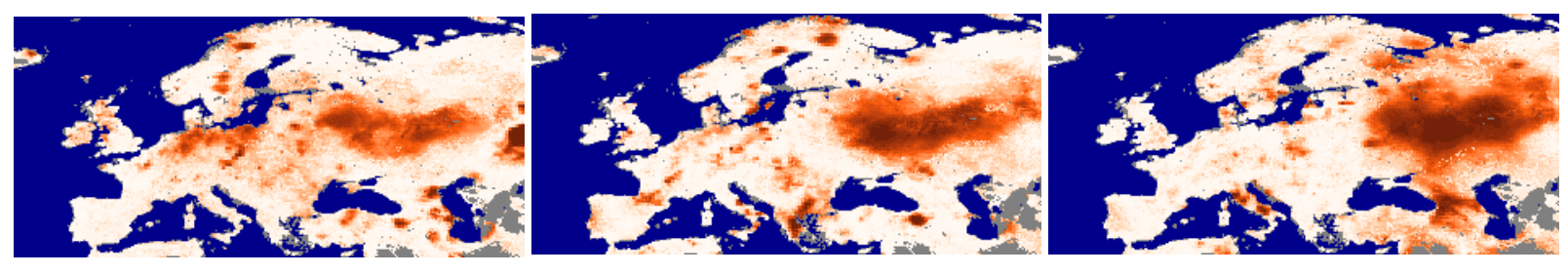

\section{m 2010-08-05}

\section{n 2010-08-13}

o 2010-08-21
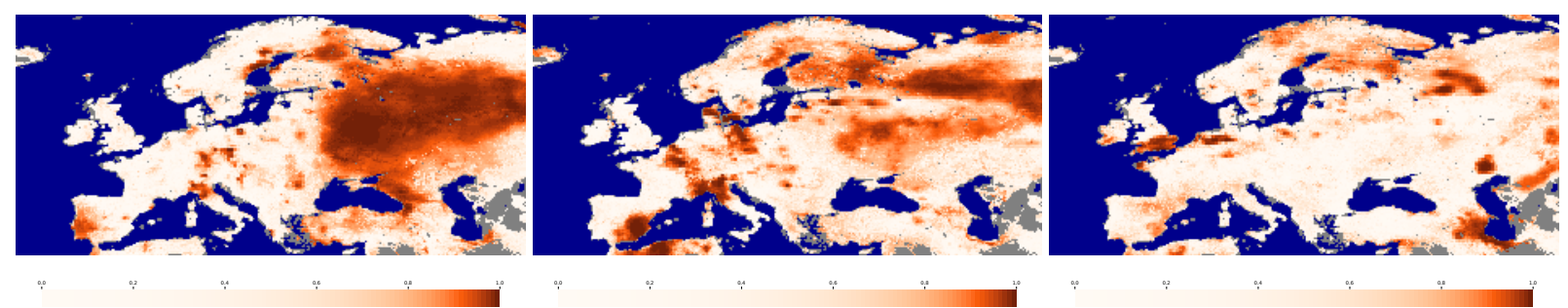

Figure S2 2. Maps of the temporal evolution of the hydrometeorological anomaly visualized as normalized ranks of the KDE. 



g 2010-06-18

h 2010-06-26

i 2010-07-04
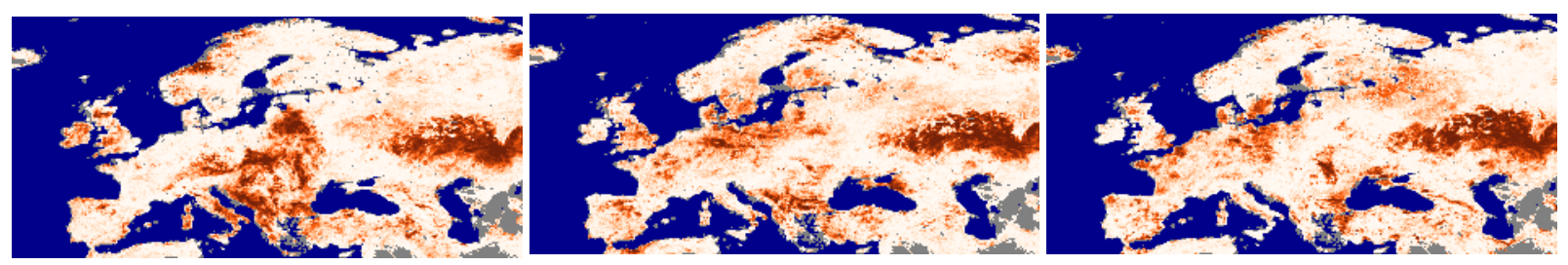

j 2010-07-12

k 2010-07-20

$12010-07-28$


m 2010-08-05

n 2010-08-13

o 2010-08-21
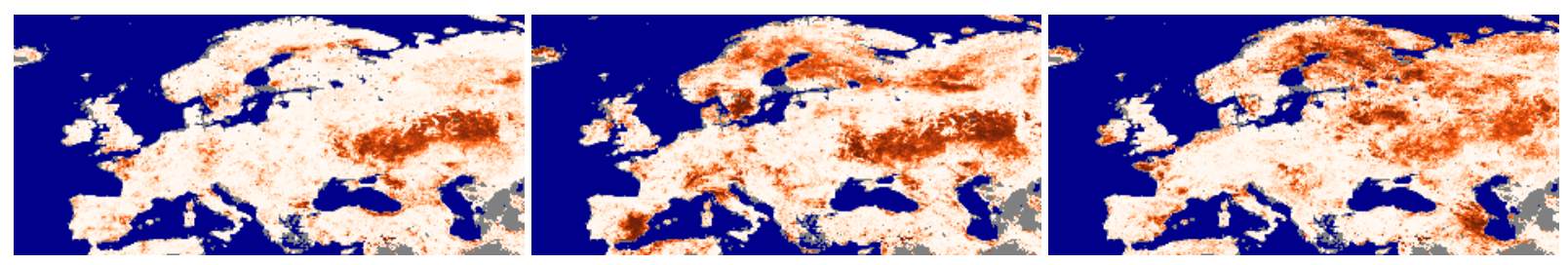

Figure S2 3. Maps of the temporal evolution of the biosphere anomaly visualized as normalized ranks of the KDE.

\section{S3 Factors explaining the GPP response}

As several factors might contribute to the GPP response to the hydrometeorological anomalies in spring in summer 2010, we assume that a linear model can partly explain the variance in GPP. The full model includes the factors temperature, precipitation, 
(a)

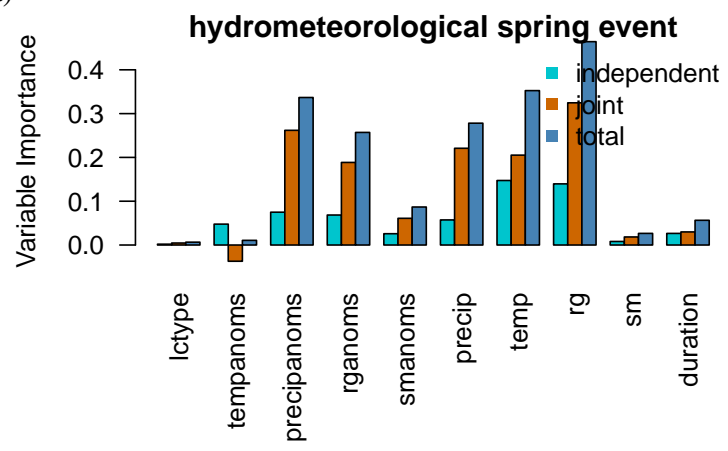

(b)

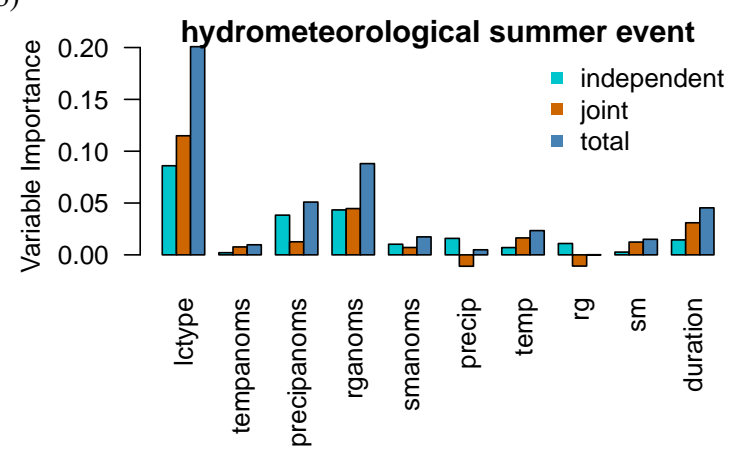

Figure S3 4. Independent, joint and total contribution of the factors explaining (a) GPP response during the hydrometeorological spring event and (b) during the hydrometeorological summer event. Used abbreviations are: vegetation type (lctype), temp (temperature), precip (precipitation), $\mathrm{rg}$ (radiation), sm (soil moisture) and their anomalies (anoms).

radiation, soil moisture, and their corresponding anomalies (spring $R^{2}=0.86$, summer $R^{2}=0.35$ ). We compute all possible combinations of submodels, and partition the variable importance into independent, joint and total contribution to the GPP response after Chevan and Sutherland (1991). Results show, that the hydrometeorological spring event is mainly a response to very favourable hydrometeorological conditions (higher radiation due to the lack of precipitation, high spring temperatures).

5 As only forest ecosystems are affected, vegetation type plays a minor role. The spring anomaly lasted only 2-3 weeks. Thus, duration is not important as well. In contrast, during the hydrometeorological summer event vegetation type is by far the most important factor explaining the GPP response, followed by radiation and precipitation anomalies and duration.

\section{S4 Sensitivity of the threshold selection}

Table S4 1. Compensation effects of the integrated hydrometeorological events (spring and summer) are not sensitive to varying the threshold for extreme event detection between $93 \%$ to $99 \%$. A slight tendency towards more pronounced compensations effects can be seen for the 90\% threshold. Such kind of enhancing the positive response is expected for lower thresholds, as the hydrometeorological conditions are not perceived as "extreme" anymore.

\begin{tabular}{lrrrrr} 
& \multicolumn{5}{c}{ Compensation [\%] } \\
Threshold & $90 \%$ & $93 \%$ & $95 \%$ & $97 \%$ & $99 \%$ \\
\hline GPP & 65 & 53 & 54 & 58 & 55 \\
NEP & 60 & 52 & 52 & 51 & 46 \\
LE & 49 & 36 & 37 & 38 & 32 \\
FAPAR & 70 & 46 & 47 & 50 & 50 \\
TER & 150 & 147 & 171 & 191 & 197
\end{tabular}




\section{S5 Coincidences}

To infer information about the state of the single variables during the multivariate extreme event, we compute the percentage of overlapping volume from the multivariate extreme as $|A \cap B| \cdot|A|^{-1}$ (sometimes also referred to as coincidences) between the hydrometeorological event (biospheric event) and the extremes in the single variables of the respective domain. For this

5 purpose we use a peak-over threshold detection scheme: anomalies in the marginal distributions of the variables are assumed to be extreme if they deviate by two standard deviations or more from the variables' mean value in the positive or negative direction.

Analysing coincidences of the marginal distributions of the variables with the multivariate biosphere event reveals insights into the state of the single variables during the biosphere anomaly. The biospheric summer event has highest coincidences with relative humidity $(81 \%)$ and surface moisture $(60 \%)$ in contrast to temperature $(14 \%)$ radiation $(20 \%)$ and precipitation $(1 \%)$ anomalies. The biospheric spring event coincides with anomalies of radiation (84\%), relative humidity (53\%) and temperature $(38 \%)$. Soil moisture is not the limiting factor during biospheric spring event $(<1 \%)$, as also reported by means of soil moistureair temperature coupling metrics (e.g., Miralles et al., 2014). These coupling metrics suggest that moisture in the higher latitudes is energy-limited during the entire positive spring anomaly and over large parts of the summer in contrast to the soil moisture

15 limited summer anomaly in the lower latitudes, enhancing sensible heat flux (Miralles et al., 2014). 


\section{References}

Chevan, A. and Sutherland, M.: Hierarchical Partitioning, The American Statistician, 45, 90-96, 1991.

Mahecha, M. D., Gans, F., Sippel, S., Donges, J. F., Kaminski, T., Metzger, S., Migliavacca, M., Papale, D., Rammig, A., and Zscheischler, J.: Detecting impacts of extreme events with ecological in situ monitoring networks, Biogeosciences, 14, 4255-4277, 2017.

5 Miralles, D. G., Teuling, A. J., van Heerwaarden, C. C., and Vilà-Guerau de Arellano, J.: Mega-heatwave temperatures due to combined soil desiccation and atmospheric heat accumulation, Nature Geoscience, 7, 345-349, 2014. 